\title{
Modification to the Centrifugation Protocol Increases the Competence of Platelet-rich Fibrin in Controlled Diabetic Patients? A Light Microscopic Study
}

\author{
Jai Abooj ${ }^{1}$, Siddhartha Varma ${ }^{2}$, Girish Suragimath ${ }^{3}$, Sameer A Zope ${ }^{4}$, Apurva Pisal ${ }^{5}$
}

\begin{abstract}
Aim: This study aims to evaluate and compare the structural characteristics of platelet-rich fibrin (PRF) prepared by two different protocols in nondiabetic and controlled diabetic patients.

Materials and methods: Ten milliliters of blood sample were drawn from 30 participants [nondiabetic (15), controlled diabetic (15)]. Five milliliters of blood were transferred to two dry vacutainers. For each patient two PRF clots were obtained using the standard protocol [leukocyte-rich platelet-rich fibrin (L-PRF)] and the low-speed concept [advanced platelet-rich fibrin (A-PRF)]. These were processed for light microscopy analysis to estimate the width of the fibrin meshwork.

Results: In controlled diabetic patients, A-PRF showed significantly more dense fibrin network compared to those of L-PRF. The difference between L-PRF and A-PRF in nondiabetic patients was not statistically significant.

Conclusion: Modification done to the standard centrifugation protocol, by increasing time and reducing speed, favors an increase in the dense fibrin network of A-PRF in diabetic patients.

Clinical significance: Modified centrifugation protocol proves to be favorable in controlled diabetic patients for periodontal regeneration.

Keywords: Controlled diabetic, Periodontitis, Platelet-rich fibrin, Regeneration.

World Journal of Dentistry (2020): 10.5005/jp-journals-10015-1693
\end{abstract}

\section{INTRODUCTION}

Periodontitis is a resultant of a deregulated inflammatory response caused by the destruction of tooth-supporting soft and hard tissues of periodontium. The major concern of the periodontal therapy is to stop the disease progression and to accomplish the vital intention of regeneration of the lost periodontal tissues. To accomplish this, various biomaterials and grafts are used, in conjunction with the conventional flap treatment.

Leukocyte-rich platelet-rich fibrin (L-PRF) is widely used in dentistry for the same purpose. Diabetes mellitus is a wellestablished risk factor for periodontitis. Delayed wound healing in diabetic patients is detrimental for regeneration. The variation in concentration of platelets in diabetic patient quantifies the efficiency of platelet-rich fibrin (PRF) in the regeneration procedure. Modifications to centrifugation, i.e., with the low-speed concept [advanced platelet-rich fibrin (A-PRF)] have shown to increase the growth factor concentrations, which has a direct effect on human gingival fibroblasts. ${ }^{1}$ Therefore, this study evaluates and compares the structural characteristics of the PRF prepared with two different protocols in nondiabetic and controlled diabetic patients for its further clinical application.

\section{Materials and Methods}

The present study was carried out in the Department of Periodontology, School of Dental Sciences, Karad after the due approval of the Ethical Committee (KIMSDU/IEC/09/2018) (0351/2018-2019). Thirty patients between 30 years and 40 years with no bleeding disorders, diagnosed with chronic generalized moderate periodontitis with their consent, were included in the study. Smokers, pregnant and lactating mothers, patients with

\footnotetext{
${ }^{1-5}$ Department of Periodontology, School of Dental Sciences, Krishna Institute of Medical Sciences (Deemed to be University), Karad, Maharashtra, India

Corresponding Author: Siddhartha Varma, Department of Periodontology, School of Dental Sciences, Krishna Institute of Medical Sciences (Deemed to be University), Karad, Maharashtra, India, Phone: +91 7620836696, e-mail: siddhartha_varma@yahoo.co.in

How to cite this article: Abooj J, Varma S, Suragimath G, et al. Modification to the Centrifugation Protocol Increases the Competence of Platelet-rich Fibrin in Controlled Diabetic Patients? A Light Microscopic Study. World J Dent 2020;11(1):65-68.

Source of support: Nil

Conflict of interest: None
}

uncontrolled diabetic or having other systemic diseases were excluded. The 30 patients were divided into two groups (15 in each group) depending on their glycemic profile. Group I consisted of nondiabetic patient and group II consisted of controlled diabetic patient. Their basic demographic data were recorded.

\section{Sample Collection}

All the aseptic precautions were followed for blood collection. Ten milliliters of intravenous blood were collected from the antecubital vein, and $5 \mathrm{~mL}$ each was immediately transferred to two disposable red top (plain) vacutainers (BD Vacutainer ${ }^{\circledR}$ ).

\section{PRF Clot Preparation}

For PRF clot preparation, these samples were centrifuged using a REMI 4 (R-4C, REMI Laboratory Instruments, Mumbai, India) 
centrifugation machine as per the protocol of Choukroun et al. For preparing the L-PRF clot, the samples were centrifuged at 2,700 rpm for 12 minutes and for the A-PRF clot at 1,300 rpm for 14 minutes. ${ }^{1}$ A sterile tweezer was used to obtain these platelet concentrate (PC) clots which were further sliced by a scissor in such a manner that only a small red blood cell (RBC) layer was conserved with rest of the clot.

\section{Histological Procedures for Light-microscopy Evaluation}

Clots were fixed in $10 \%$ formalin for 72 hours. Then, they were transferred into a perforated stainless steel cassette with a label placed inside for identification. Next, the clots were subjected to dehydration by passing through various processing solutions such as $60 \%, 70 \%, 80 \%, 90 \%$, and $100 \%$ isopropanol alcohol. Then, they were placed in the clearing solution, xylene (two changes), followed by infiltration of paraffin wax. Tissue sectioning was done on microtome (Leica Biosystems) followed by dewaxing of slides. Routine hematoxylin and eosin (Loba Chemie) staining was performed. The slides were then numbered for records and viewed under a light microscope (MagnAüus Theia) under 10× and $40 \times$ magnification. Images were captured and subjected to image analysis with the use of auto CAD software (Autodesk, version 2016) for the evaluation of width of the dense fibrin network and the loose fibrin network along with the enmeshment pattern of platelets and white blood cells (WBCs) within the network for both the groups.

\section{Statistical Analysis}

Data were analyzed by the Tukey-Kramer comparison test by SPSS software version 20.0. Demographic data were evaluated using Student's $t$ test. $p$ value of $<0.05$ was considered to be statistically significant for both the tests.

\section{Results}

On histological evaluation, it was observed that the samples from both the groups had an outermost layer of RBCs, a dense fibrin network layer which contained a maximum number of platelets and WBCs entrapped in it followed by a loose fibrin network pattern with reduced entrapment of platelets and WBCs as shown in Figures 1 to 4.

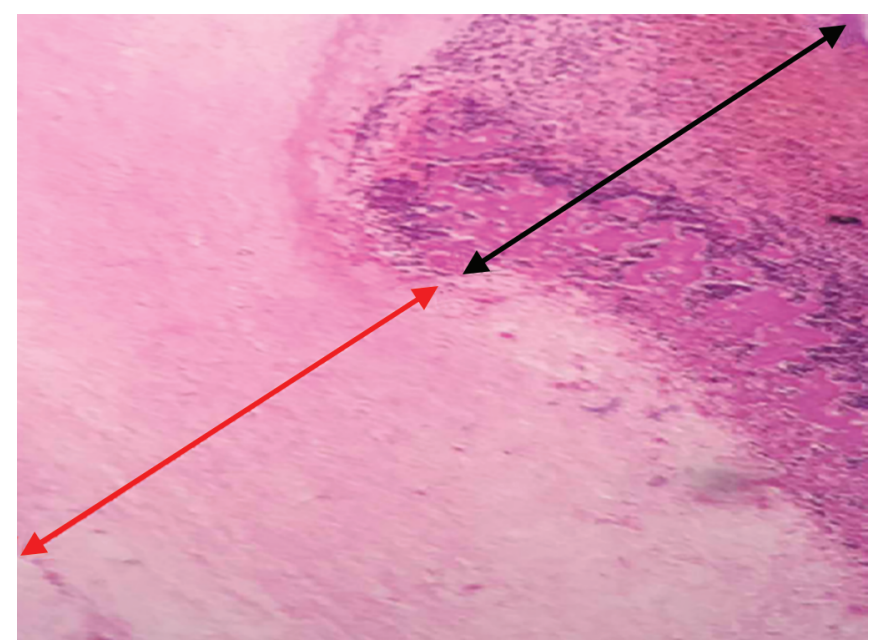

Fig. 1: Group I (nondiabetic group with chronic periodontitis) advanced platelet-rich fibrin. Black marking denotes the dense fibrin network and red denotes the loose fibrin network. Under 40x
Figure 5 represents the percentage of dense and loose fibrin network patterns in both the groups. It was found that there was sufficient dense fibrin network in the A-PRF clot (45\%) as compared to the L-PRF clot (35\%) in group II. This difference was found to be statistically significant ( $p$ value $=0.0044$ ).

In group I, the A-PRF clot was found to have slightly more amount of dense fibrin network (55\%) than the L-PRF clot (52\%), but the difference was not statistically significant.

Upon comparing the enmeshment pattern of platelets and WBCs within the dense and loose fibrin networks, it was found that in group I (nondiabetic group) more cells were layered within the dense fibrin network, whereas in the loose fibrin network few cells were diffusely arranged. In group II (controlled diabetic group), platelet and WBC were more at the periphery of the dense fibrin network. The quantity was also reduced in L-PRF as compared to A-PRF.

There was no statistical difference found in the basic demographic data of both the groups represented in Table 1.

\section{Discussion}

The aim of this study was to evaluate and compare the structural characteristics of PRF prepared with different protocols in nondiabetic and controlled diabetic patients. As the chronic hyperglycemic profile of the diabetic patients tends to result in various complications. ${ }^{2}$ One such complication is the altered healing which leads to the unfavorable results of periodontal treatment procedures. This becomes a major concern for regenerative therapy in such patients.

The concept of using PCs as biomaterials for accelerated healing dates back to 1970s when the Fibrin glue was first described. ${ }^{3}$ It was found to be least favorable for cytokine enmeshment and cellular migration. In 1979, "gelatin platelet-gel foam" or "plateletfibrinogen-thrombin mixtures" were used chiefly for their "gluey effect". Its application in accelerating healing and release of growth factors were not considered yet. ${ }^{4}$ Knighton et al. in 1986 reported that PCs were successful in promoting healing and termed it as platelet-derived wound healing factors. ${ }^{5}$ The first-generation PC was introduced in 1988 by Marx et al. and was termed as plateletrich plasma (PRP). ${ }^{6}$ It was and is still being utilized in various fields but it contains anticoagulants, which prevent the full coagulation cascade essential for tissue wound healing. With the introduction of the L-PRF in 2001 by Choukroun et al. it is now widely used as a low-cost fibrin matrix for tissue regeneration. ${ }^{7}$ No anticoagulants

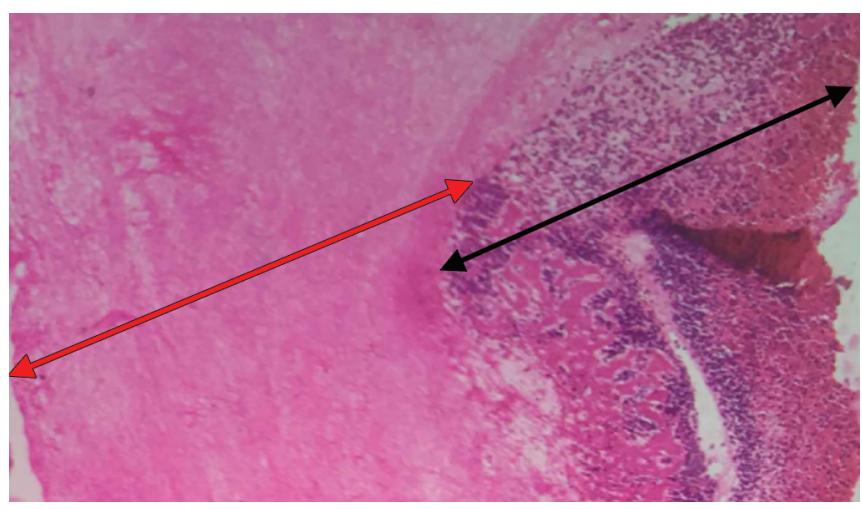

Fig. 2: Group I (nondiabetic group with chronic periodontitis) leukocyterich platelet-rich fibrin. Black marking denotes the dense fibrin network and red denotes the loose fibrin network. Under 40x 


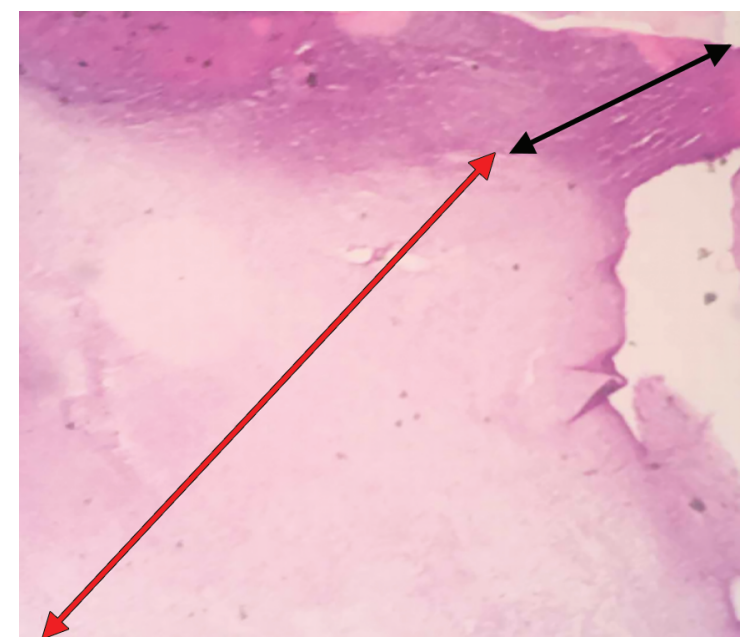

Fig. 3: Group II (controlled diabetic group with chronic periodontitis) leukocyte-rich platelet-rich fibrin. Black marking denotes the dense fibrin network and red denotes the loose fibrin network. Under 40x

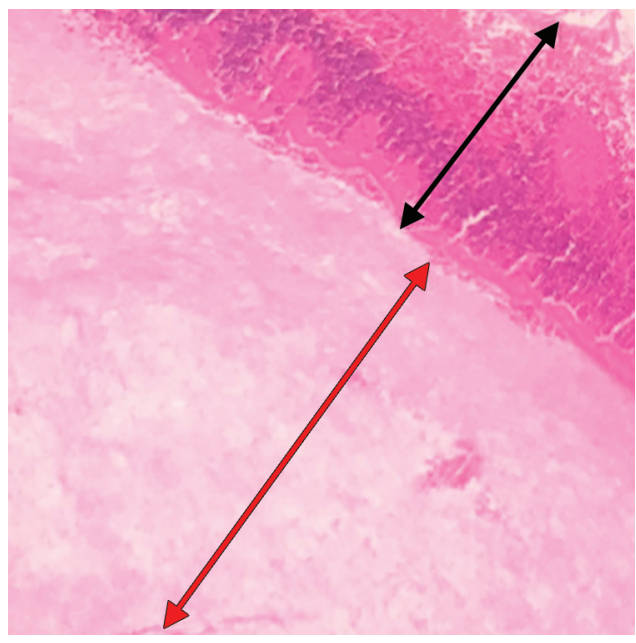

Fig. 4: Group II (controlled diabetic group with chronic periodontitis) advanced platelet-rich fibrin. Black marking denotes the dense fibrin network and red denotes the loose fibrin network. Under 40x

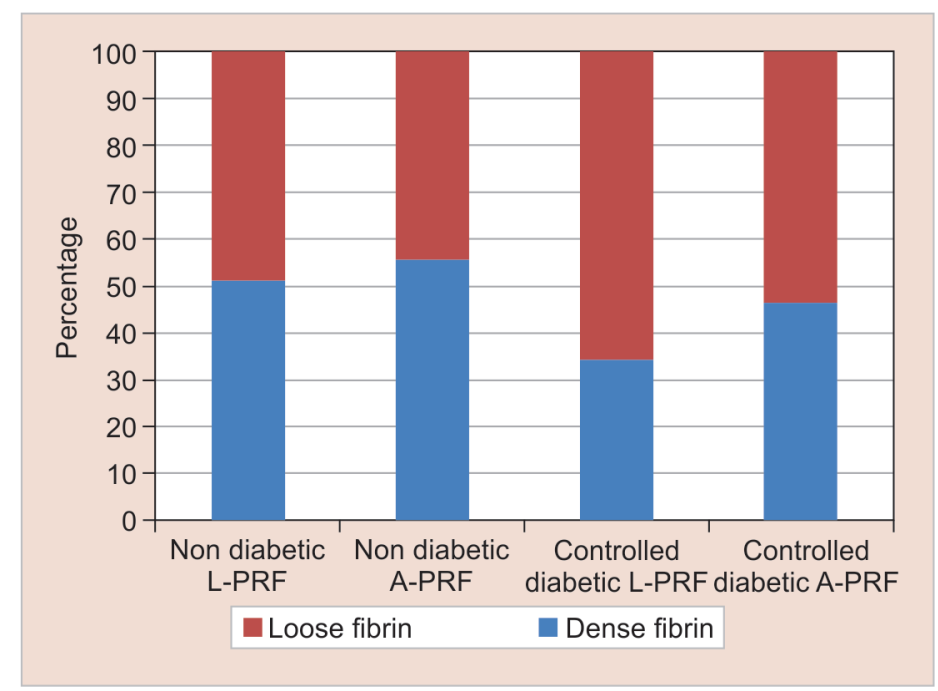

Fig. 5: Comparison of fibrin network pattern in both the groups

Table 1: Comparison of demographic data of both groups

\begin{tabular}{llrl}
\hline Parameters & Group & Mean \pm SD & \multicolumn{1}{l}{ p value } \\
\hline Age & Group I & $35.133 \pm 3.2$ & $0.91(\mathrm{NS})^{\mathrm{a}}$ \\
& Group II & $35.266 \pm 3.2$ & \\
\multirow{3}{*}{ Gender } & Group I & $1.4 \pm 0.5$ & $0.77(\mathrm{NS})^{\mathrm{a}}$ \\
& Group II & $1.4 \pm 0.5$ & \\
\hline
\end{tabular}

$\mathrm{SD}$, standard deviation; NS, statistically nonsignificant

astudent's $t$ test

are required for PRF preparation, thus it overcomes the drawback of PRP. Another advantage of PRF is its three-dimensional fibrin matrix structure which can act as a scaffold in different procedures, like in cases of guided bone and tissue regeneration it works as a barrier membrane. , $^{8}$

It must be understood that the success of PRF depends on the concentration of platelets which determines its efficiency for regeneration. The cytokines released on degranulation of platelets encourage the cell migration and proliferation within the fibrin matrix, thus upregulating the first stages of healing. In diabetic patients altered polymorphonuclear leukocyte function, collagen metabolism and impaired chemotaxis along with altered PCs make the treatment unpredictable. ${ }^{10}$

Choukroun et al. and Dohan et al. observed that the concentration of platelets was maximum in the buffy coat. ${ }^{11,12}$ Laurens et al. observed that the fibrin structure of PRF, for example, the thickness of the fibers influences the wound healing significantly. Apart from it, PCs and their functions are also the influencers. ${ }^{13}$ Thus, in this study, the buffy coat region of the PRF was histologically evaluated and compared for its network pattern along with the distribution of platelets and WBC in both the groups.

In this study, it was observed that there was a significant increase in the dense fibrin network in group II (controlled diabetic group) on using the A-PRF protocol as compared to L-PRF. This indicated that the low-speed concept has favorable results. These results are in accordance with the study carried out by Ghanaati et al. in which a large number of leukocytes were found in the vicinity of the bottom part of the fibrin clot in L-PRF as compared to A-PRF where it was found more in the distal part. ${ }^{14}$ 
Fujioka-Kobayashi et al. found that A-PRF prepared by the modified centrifugation protocol increased the growth factor concentrations which directly affects the human gingival fibroblasts. ${ }^{1}$

The PRFs obtained from different age groups were evaluated for variations in fibrin network patterns by Yajamanya et al. It was found that there was a significant decrease in the dense fibrin network and increase in the loose fibrin network as the age progressed. Alterations in the number of cells entrapped in the fibrin network were also noticed..$^{15}$ In this study, the age group selected for both the groups was $30-40$ years, with no statistical difference, thus, chances of variation with age were absent.

This study highlights the outcome of the modified centrifugation protocol on fibrin clot formation and its interaction of cellular component in controlled diabetic patients. In this study, hematoxylin and eosin stain and light microscopic evaluation were used which proved the satisfactory results in identifying the variation in the fibrin network pattern and the enmeshment pattern of platelets and WBCs within it. But it was hard to recognize and separate platelets and WBCs from each other. Hence, to state the precise number of platelets trapped within fibrin was also difficult. Further studies with a larger sample size and better staining and scanning techniques are required to overcome this limitation.

\section{Conclusion}

To conclude, this study demonstrates that modifications to the centrifugation protocol by decreasing the speed and increasing the time have favorable results as it increases the dense fibrin network of A-PRF as compared to L-PRF in controlled diabetic patients. Advanced PRF showed better structural characteristics than L-PRF in controlled diabetic patients. This needs to be further confirmed with long-term clinical studies.

\section{References}

1. Fujioka-Kobayashi M, Miron RJ, Hernandez M, et al. Optimized platelet-rich fibrin with the low-speed concept: growth factor release, biocompatibility, and cellular response. J Periodontol 2017;88(1):112121. DOI: 10.1902/jop.2016.160443.

2. Verhulst MJ, Loos BG, Gerdes VE, et al. Evaluating all potential oral complications of diabetes mellitus. Front Endocrinol (Lausanne) 2019;10:56. DOI: 10.3389/fendo.2019.00056.
3. Sunitha Raja V, Munirathnam Naidu E. Platelet-rich fibrin: evolution of a second-generation platelet concentrate. Indian J Dent Res 2008;19(1):42-46. DOI: 10.4103/0970-9290.38931.

4. Rosenthal AR, Egbert PR, Harbury C, et al. Use of platelet-fibrinogenthrombin mixture to seal experimental penetrating corneal wounds. Albrecht Von Graefes Arch Klin Exp Ophthalmol 1978;207(2):111-115. DOI: $10.1007 / B F 00414308$.

5. Knighton DR, Ciresi KF, Fiegel VD, et al. Classification and treatment of chronic nonhealing wounds. successful treatment with autologous platelet-derived wound healing factors (PDWHF). Ann Surg 1986;204(3):322-330. DOI: 10.1097/00000658-19860900000011.

6. Marx RE, Carlson ER, Eichstaedt RM, et al. Platelet-rich plasma: growth factor enhancement for bone grafts. Oral Surg Oral Med Oral Pathol Oral Radiol Endod 1998;85(6):638-646. DOI: 10.1016/ S1079-2104(98)90029-4.

7. Choukroun J, Adda F, Schoeffer C, et al. PRF: an opportunity in perio implantology. Implantodontie 2000;42:55-62.

8. Agrawal AA. Evolution, current status and advances in application of platelet concentrate in periodontics and implantology. World J Clin cases 2017;16(5):159-171. DOI: 10.12998/wjcc.v5.i5.159.

9. Toffler M. Guided bone regeneration (GBR) using cortical bone pins in combination with leukocyte-and platelet-rich fibrin (L-PRF). Compend Contin Educ Dent 2014;35(3):192-198.

10. Mealey BL. Periodontal disease and diabetes: a two-way street. J Am Dent Assoc 2006;137(Suppl):26-31. DOI: 10.14219/jada. archive.2006.0404.

11. Choukroun J, Diss A, Simonpieri A, et al. Platelet-rich fibrin (PRF): a second-generation platelet concentrate. part IV: clinical effects on tissue healing. Oral Surg Oral Med Oral Pathol Oral Radiol Endod 2006;101(3):e56-e60. DOI: 10.1016/j.tripleo.2005.07.011.

12. Dohan DM, Choukroun J, Diss A, et al. Platelet-rich fibrin (PRF): a second-generation platelet concentrate. part II: platelet-related biologic features. Oral Surg Oral Med Oral Pathol Oral Radiol Endod 2006;101(3):45-50. DOI: 10.1016/j.tripleo.2005.07.009.

13. Laurens N, Koolwijk P, de Maat MP. Fibrin structure and wound healing. J Thromb Haemost 2006;4(5):932-939. DOI: 10.1111/j.15387836.2006.01861.x.

14. Ghanaati S, Booms P, Orlowska A, et al. Advanced platelet-rich fibrin: a new concept for cell-based tissue engineering by means of inflammatory cells. J Oral Implantol 2014;40(6):679-689. DOI: 10.1563/ aaid-joi-D-14-00138.

15. Yajamanya SR, Chatterjee A, Babu CN, et al. Fibrin network pattern changes of platelet-rich fibrin in young vs old age group of individuals: a cell block cytology study. J Indian Soc Periodontol 2016;20(2):151. DOI: 10.4103/0972-124X.176390. 